\title{
Pre-flight qualification tests of the Mini-EUSO telescope engineering model
}

\author{
F. Bisconti ${ }^{1,2}$ (D) $\cdot$ H. Miyamoto ${ }^{1,2} \cdot$ D. Barghini ${ }^{1,2,3}$ (D) M. Battisti, ${ }^{1,2}$ (D) A. Belov . $^{4}$ \\ M. E. Bertaina ${ }^{1,2}$ (D) S. Blin-Bondil ${ }^{5,15}$. G. Cambièe ${ }^{6,7} \cdot$ F. Capel ${ }^{8,9}$. \\ M. Casolino $6,7,10 \cdot$ A. Cellino ${ }^{2,3} \cdot$ L. Conti $^{6,11}$. G. Contino ${ }^{12}$ (D) . G. Cotto ${ }^{1,2}$. \\ T. Ebisuzaki ${ }^{10} \cdot$ F. Fenu ${ }^{1,2} \cdot$ C. Fornaro ${ }^{11}$ - A. Franceschi ${ }^{13}$. D. Gardiol ${ }^{3}$ (D) . \\ A. Haungs ${ }^{14}$ (D) P. Klimov ${ }^{4}$ (D) M. Manfrin ${ }^{1,2} \cdot$ L. Marcelli $^{6} \cdot$ M. Mignone ${ }^{2}$. \\ T. Napolitano ${ }^{13}$. E. Parizot ${ }^{15}$ (D) . P. Picozza ${ }^{6,7}$. L. W. Piotrowski ${ }^{16,10}$. \\ G. Prévôt ${ }^{15} \cdot$ E. Reali ${ }^{6,7} \cdot$ M. Ricci ${ }^{13}$ (D) K. Shinozaki ${ }^{1,17} \cdot$ F. Simioli ${ }^{11} \cdot$ G. Suino ${ }^{1,2}$. \\ J. Szabelski ${ }^{17}$
}

Received: 14 June 2021 / Accepted: 29 September 2021 / Published online: 20 October 2021

(C) The Author(s) 2021

\begin{abstract}
Mini-EUSO is part of the JEM-EUSO program and operates on board the International Space Station (ISS). It is a UV-telescope with single-photon counting capability looking at nighttime downwards to the Earth through a nadir-facing UVtransparent window. As part of the pre-flight tests, the Mini-EUSO engineering model, a telescope with 1/9 of the original focal surface and a lens of $2.5 \mathrm{~cm}$ diameter, has been built and tested. Tests of the Mini-EUSO engineering model have been made in laboratory and in open-sky conditions. Laboratory tests have been performed at the TurLab facility, located at the Physics Department of the University of Turin, equipped with a rotating tank containing different types of materials and light sources. In this way, the configuration for the observation of the Earth from space was emulated, including the Mini-EUSO trigger schemes. In addition to the qualification and calibration tests, the Mini-EUSO engineering model has also been used to evaluate the possibility of using a JEM-EUSO-type detector for applications such as observation of space debris. Furthermore, observations in open-sky conditions allowed the studies of natural light sources such as stars, meteors, planets, and artificial light sources such as airplanes, satellites reflecting the sunlight, and city lights. Most of these targets could be detected also with Mini-EUSO. In this paper, the tests in laboratory and in open-sky conditions are reported, as well as the obtained results. In addition, the contribution that such tests provided to foresee and improve the performance of Mini-EUSO on board the ISS is discussed.
\end{abstract}

F. Bisconti

francesca.bisconti@to.infn.it

Extended author information available on the last page of the article. 
Keywords Mini-EUSO · Fluorescence detector · Near-UV observations · EUSO@TurLab·Stars · Meteors

\section{Introduction}

Mini-EUSO [1] is the first space-based mission of the JEM-EUSO (Joint Experiment Missions for Extreme Universe Space Observatory) program [2], installed inside the International Space Station (ISS). The JEM-EUSO program is devoted to detect Ultra-High Energy Cosmic Rays (UHECRs) from space, through the fluorescence detection technique. When charged particles in extensive air showers induced by primary cosmic rays excite nitrogen molecules in the atmosphere, the molecular fluorescence transition leads to the isotropic light emission in the near-UV band (290-430 nm), which can be detected with highly sensitive telescopes at nighttime. Moreover, fluorescence detectors observe also the Cherenkov light emitted when charged particles travel faster than the speed of light in that medium. The Cherenkov emission occurs along the shower axis and can be observed directly, measuring photons coming from the characteristic Cherenkov cone (by ground-based detectors), or after ground reflection (by high-altitude detectors).

A detector on satellite would be able to observe the extensive air shower along its development in the atmosphere. Moreover, orbiting around the Earth it would have a full-sky coverage, allowing the direct study of large scale anisotropies and the search for sources of UHECRs, as they are not significantly deflected by magnetic fields. With a wide field of view, such a detector can increase the probability to detect cosmic rays at the extreme energies with respect to ground-based observatories. In addition, a space-based observatory allows to study a variety of atmospheric phenomena including transient luminous events (sprites, blue jets, elves, etc.), and lightning discharges, as well as meteors and, possibly, samples of strange quark matter (nuclearites) [3]. Ground-based light sources like anthropogenic lights and bioluminescence can also be observed.

To prove the UHECRs detection principle and study the aforementioned phenomena and their possible impact on the detection of UHECRs from space, the Mini-EUSO mission has been developed as a joint project between the Italian (ASI) and Russian (Roscosmos) space agencies. Mini-EUSO has been installed inside the ISS in August 2019, at an altitude of $\sim 400 \mathrm{~km}$ on the UV transparent window in the Russian Zvezda module. It is active since October 2019, with some data acquisition sessions being performed each month.

Mini-EUSO and its main science objectives are depicted in Fig. 1. Among the other objectives already described for a space-based telescope, Mini-EUSO will provide the mapping of the Earth in the near-UV band at night, giving important information on the emissions from the Earth's surface, which will also represent the background for the detection of other phenomena occurring in the atmosphere. Although the present configuration of Mini-EUSO on the ISS is not optimal for the detection of orbiting space debris, it is important to study the capability of the JEMEUSO-like telescopes [4] to detect and track them. For their high temporal sampling rates, this type of telescopes are under consideration for the development of a system 


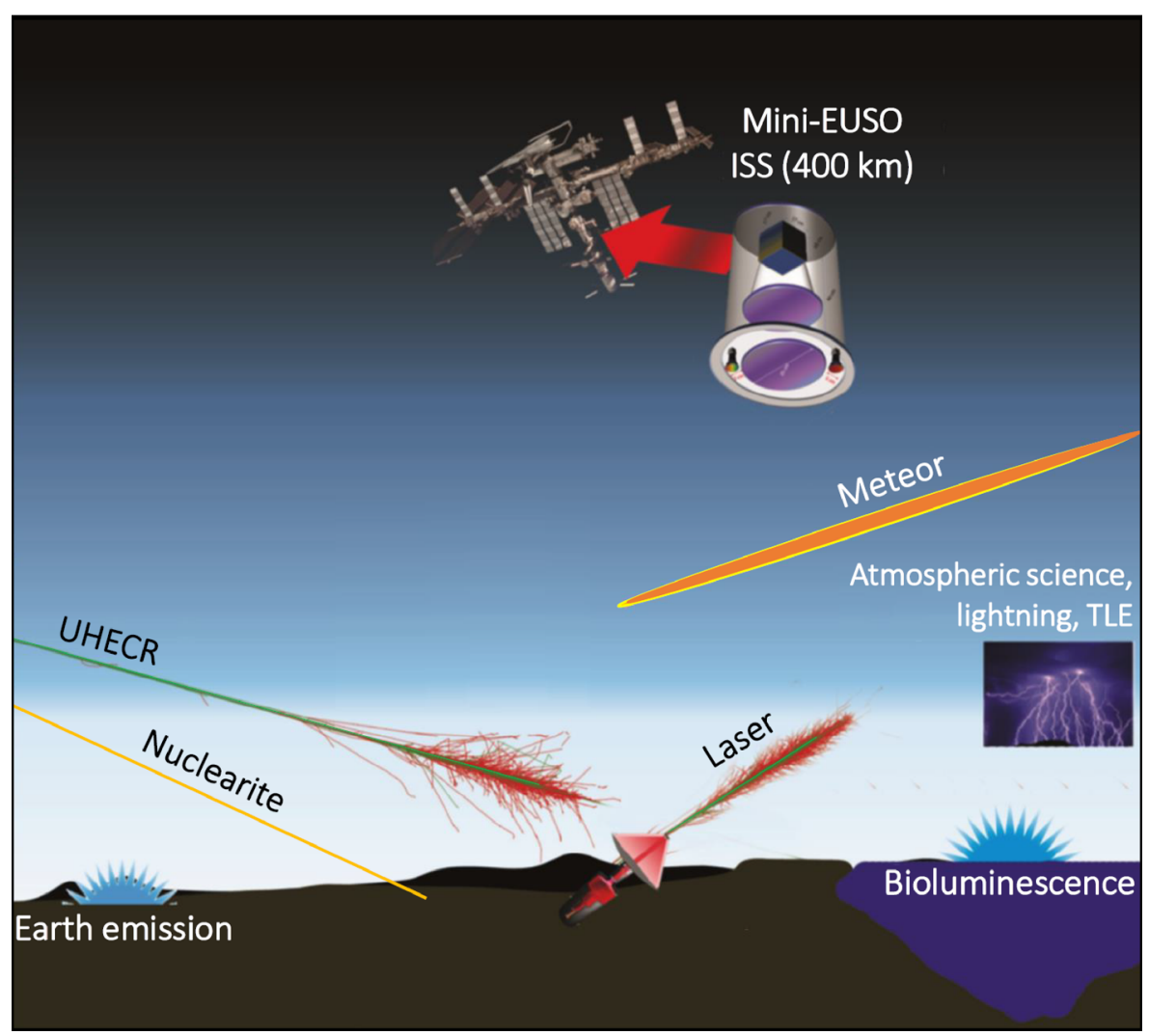

Fig. 1 The Mini-EUSO mission with several of its target light sources: emissions from the Earth (for example cities), from the sea (bioluminescence), meteors, candidate events of strange quark matter (nuclearites), atmospheric events, UHECRs, and scattered light from laser emissions

on a free-flyer satellite for space debris detection, tracking and, in combination with a high-energy laser, possible remediation [5]. To calibrate Mini-EUSO in its final stage on board the ISS, ground-based lasers shooting in the field of view of the telescope will be used [6-8].

A Mini-EUSO engineering model (Mini-EUSO EM) was built and tested. It was used in the qualification tests (vibration, electric and electromagnetic interference and compatibility, and thermal-vacuum/environmental tests), with lens mock-up (without the Fresnel structure). The flight model underwent electric and vibration tests at a reduced level [1]. The performance of the Mini-EUSO EM have been verified at the Physics Department of the University of Turin and at the Astrophysical Observatory of Turin, in Italy, in February and March 2018. At the Physics Department, laboratory tests have been made at the TurLab facility [9] that hosts a rotating tank used to perform analysis with moving light sources. Outdoor observations have been made from the roof of the building, pointing the telescope to flashers and lit buildings. Stars, a few meteor candidates, and a large-scale space debris (a rocket 
body) have been observed from the Astrophysical Observatory, where the sky conditions allowed observation of faint sources, as well as artificial lights from nearby cities. These measurements and related results are presented and discussed in this paper.

\section{Detector setup and data acquisitions}

\subsection{Mini-EUSO}

The Mini-EUSO detector [1] has an optical system composed of two Fresnel lenses of $25 \mathrm{~cm}$ diameter and $20.5 \mathrm{~cm}$ focal length, made of Poly(methyl methacrylate) - PMMA. Photons are focused on a focal surface with $6 \times 6$ Multi-Anode PhotoMultiplier Tubes (MAPMTs) [10], with $8 \times 8$ pixels of $2.88 \mathrm{~mm}$ side, for a total of 2304 pixels. The MAPMTs are grouped in 9 Elementary Cells (ECs), made of $2 \times$ 2 MAPMTs and the front-end electronics. The system of 9 ECs represents the PhotoDetector Module (PDM). The sensitivity band is the near-UV, thanks to a $2 \mathrm{~mm}$ thick UV band-pass filter made of BG3 material and with anti-reflective coating. Filters are glued on each single MAPMT, and allow photons in the wavelength range $\sim 290-430 \mathrm{~nm}$ to reach the sensors. Pictures in Fig. 2 show the Mini-EUSO detector components, with the external Fresnel lens in the panel (a) and the complete focal surface with $9 \mathrm{ECs}$, i.e. 36 MAPMTs, in the panel (b). The focal surface and the optical system let the detector have a square field of view of $\sim 44^{\circ} \times 44^{\circ}$, allowing for observations of the Earth with a spatial resolution of $\sim 6 \mathrm{~km}$ projected on the ground. Each EC consists of 4 MAPMTs with independent EC-Anode board and a common single high voltage power supply with EC-Dynode distribution board. Signals are pre-amplified and digitized by one 64-channel ASIC chip (SPACIROC3) [11] per MAPMT. Gaps present between MAPMTs of the same EC and between different ECs are required by mechanical fixations and electric insulation.

The telescope has single-photon counting capability. The data are sampled in Gate Time Units (GTUs) $2.5 \mu \mathrm{s}$ long, and saved with three time resolutions, to cover the different timescales typical for the various phenomena that Mini-EUSO aims to observe. Namely, D1 GTU refers to the aforementioned GTU $(2.5 \mu \mathrm{s})$, D2 GTU refers to $128 \times 2.5 \mu \mathrm{s}(320 \mu \mathrm{s})$, and D3 GTU corresponds to $128 \times 128 \times 2.5 \mu \mathrm{s}$ (40.96 ms). For each pixel, the counts collected in each single D2 and D3 GTU correspond to the sum of counts collected over the corresponding number of D1 GTUs (128 for D2 GTUs and $128 \times 128$ for D3 GTUs). The D1 and D2 timescale events have dedicated trigger logic devoted to their detection, L1 and L2 trigger respectively $[12,13]$, while a continuous acquisition of the signal integrated over D3 GTUs is performed. The L1 trigger analyzes data with a time resolution of 1 D1 GTU and looks for signal excess on a timescale of $20 \mu \mathrm{s}$ (8 D1 GTUs). This is the typical timescale of cosmic-ray-like events, also well suitable for the detection of elves and flashers with timescales of $\sim 0.01-1 \mathrm{~ms}$ [1]. Data integrated over 128 D1 GTUs are used to determine the background level and, at the same time, they are passed to the L2 trigger logic, well-suited to capturing atmospheric events such as lightning with timescales of $\sim 1-100 \mathrm{~ms}$ [1]. Packets of 128 D1 GTUs are stored as D2 data in case 


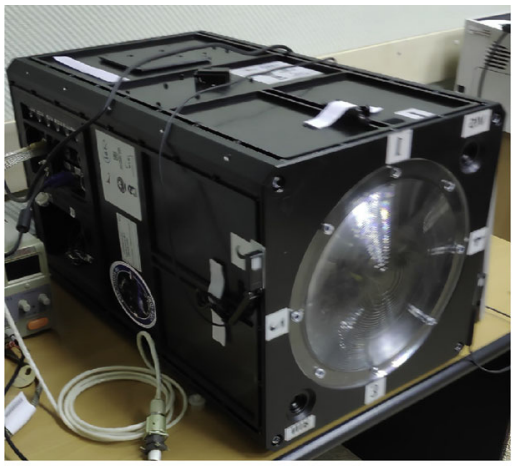

(a)

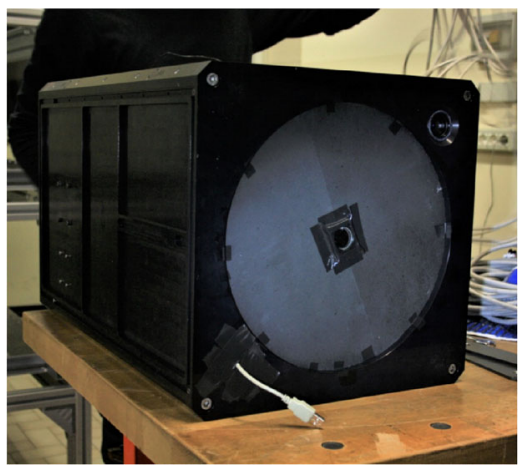

(c)

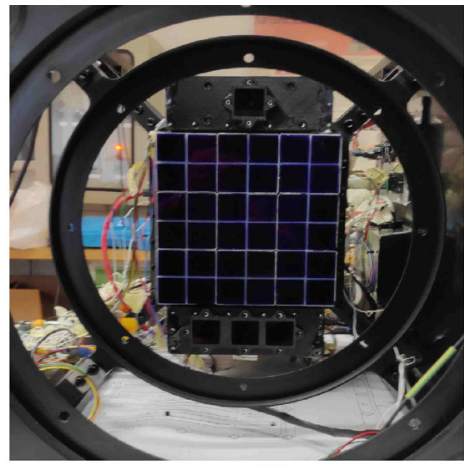

(b)

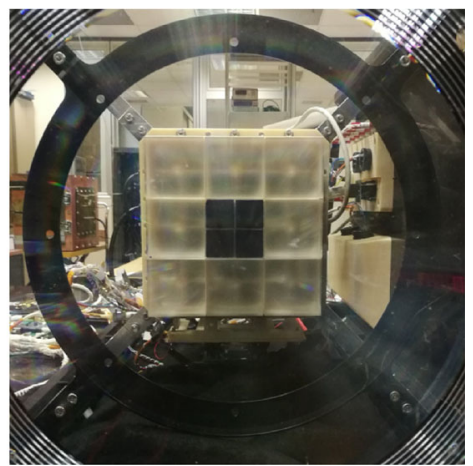

(d)

Fig. 2 (a) Mini-EUSO exterior view, with the front Fresnel lens of $25 \mathrm{~cm}$ diameter; (b) its internal focal surface made of $6 \times 6$ MAPMTs. (c) Mini-EUSO EM exterior view, with the lens of $2.5 \mathrm{~cm}$ diameter; (d) its internal focal surface made of $2 \times 2$ MAPMTs. Photos of Mini-EUSO taken from [1]

of L2 triggers. A continuous acquisition over $128 \times 128$ D1 GTUs is also stored as D3 data. During the further accumulation of 128 D3 GTUs (5.24 s in total) up to four events detected with the L1 and L2 triggers can be stored and transferred to the CPU together with the D3 data, for formatting and storage on the disk. In this way, a continuous readout is achieved with a resolution of $40.96 \mathrm{~ms}$ whilst also recording interesting events at shorter timescales. Data frames integrated over D3 GTUs are used to search for meteors, space debris and strange quark matter, as well as for the mapping of the Earth in the near-UV.

\subsection{The Mini-EUSO EM}

The Mini-EUSO EM is a detector similar to the Mini-EUSO flight model but with a plano-convex lens of $2.5 \mathrm{~cm}$ diameter and $30 \mathrm{~cm}$ focal length as optical system, and a focal surface corresponding to the central EC of the Mini-EUSO focal surface. The decision of using a different lens system with respect to Mini-EUSO was made to avoid the risk of damaging the original Fresnel lenses. Since the main goal of the 
data acquisitions was to test the performance of the electronics, the loss in terms of light collection caused negligible consequences. Also the Mini-EUSO EM has a square field of view but it is $\sim 10^{\circ} \times 10^{\circ}$, due to the reduced focal surface and the different optics. In Fig. 2 the exterior of the Mini-EUSO EM is shown in the panel (c), including the lens at the center of the front side, while its focal surface is shown in the panel (d), composed only of the central EC.

The read-out system and the trigger logic used for the Mini-EUSO EM are the same as those for the flight model. Therefore, data are sampled on three timescales with resolution of $2.5 \mu \mathrm{s}, 320 \mu \mathrm{s}$ and $40.96 \mathrm{~ms}$.

\subsection{Flight model vs. engineering model of Mini-EUSO}

In the previous subsections, technical information on the Mini-EUSO EM and MiniEUSO has been reported. Here, a comparison of their performance is provided, based on the difference between the two setups. Table 1 summarizes the comparison of key specifications of the engineering and flight models.

As the lens size of the Mini-EUSO EM is 1/10 of that of Mini-EUSO, the former is $1 / 100$ times as efficient as the latter (to collect the light). The table contains also the focal ratio, defined as the ratio of the focal length to the diameter of the aperture, a parameter usually adopted for optical systems to define their sensitivity.

The field of view of the Mini-EUSO EM $\left(\sim 10^{\circ} \times 10^{\circ}\right)$ was estimated from the position of stars detected during outdoor measurements. Instead, that of Mini-EUSO $\left(\sim 44^{\circ} \times 44^{\circ}\right)$ was formerly estimated with simulations during the designing phase, then confirmed with measurements from space using cities as reference. These values include the gaps between the MAPMTs. The field of view of one pixel is $\sim 0.6^{\circ}$ $\times 0.6^{\circ}$ for the Mini-EUSO EM and $\sim 0.8^{\circ} \times 0.8^{\circ}$ for Mini-EUSO. As the solid angle seen by one pixel of the Mini-EUSO EM is approximately half that of Mini-EUSO $\left(0.6^{2} / 0.8^{2} \simeq 0.6\right)$, the amount of background light collected by one pixel of the former detector is about $1 / 200$ the background collected by the latter (considering both the contribution of the optics and the pixel field of view).

Table 1 Comparison of the key specifications of Mini-EUSO EM and Mini-EUSO

\begin{tabular}{lll}
\hline & Mini-EUSO EM & Mini-EUSO \\
\hline Focal Surface & $2 \times 2$ MAPMTs & $6 \times 6$ MAPMTs \\
& $16 \times 16=256$ pixels & $48 \times 48=2304$ pixels \\
Optical system & 1 plano-convex lens & 2 Fresnel lenses \\
Lens(-es) diameter & $2.5 \mathrm{~cm}$ & $25 \mathrm{~cm}$ \\
Focal length & $30 \mathrm{~cm}$ & $20.5 \mathrm{~cm}$ \\
Focal ratio & $f / 12$ & $f / 0.82$ \\
Field of view & square & square \\
Total (including gaps) & $\sim 10^{\circ} \times 10^{\circ}$ & $\sim 44^{\circ} \times 44^{\circ}$ \\
Pixel & $\sim 0.6^{\circ} \times 0.6^{\circ}$ & $\sim 0.8^{\circ} \times 0.8^{\circ}$ \\
\hline
\end{tabular}


The signal-to-noise ratio for the sensitivity to point-like sources is proportional to the amount of signal from the source reaching a pixel and inversely proportional to the square root of the amount of background, which is determined by the solid angle seen by a pixel. Therefore, the signal-to-noise ratio of the Mini-EUSO EM is $\sim 0.01 / \sqrt{(0.01 / 2)} \simeq 1 / 7$ times that of Mini-EUSO. This estimation is made by taking into account the same background intensity and the same light source apparent intensity.

\section{Tests at TurLab}

\subsection{TurLab and the EUSO@TurLab project}

TurLab is a facility designed for geo-fluid-dynamics studies. It hosts a tank of $5 \mathrm{~m}$ diameter that has the capability of rotating at speeds ranging from $3 \mathrm{~s}$ to $600 \mathrm{~s}$ per rotation. Moreover, as it is located in a very dark environment, the intensity of background light can be adjusted in a controlled condition.

A series of measurement campaigns devoted to test several prototypes and pathfinders of the JEM-EUSO program have been performed at TurLab [14, 15]. These telescopes are designed to observe the Earth's atmosphere from the stratosphere or from space. By hanging the telescope on the ceiling above the rotating tank (see Fig. 3), a detector in orbit observing the Earth was mimicked. In this environment, the performance of the electronics and the L1 and L2 trigger logic was tested, as well as the response to atmospheric phenomena such as meteors and lightning reproduced in the laboratory.

\subsection{The Mini-EUSO EM at TurLab}

In February and March 2018, the Mini-EUSO EM was tested at TurLab. After preliminary operation tests at its arrival, it was hung on the ceiling above the TurLab tank to be tested for its general performance as well as for the data acquisition and control software [12] and the trigger system [13].
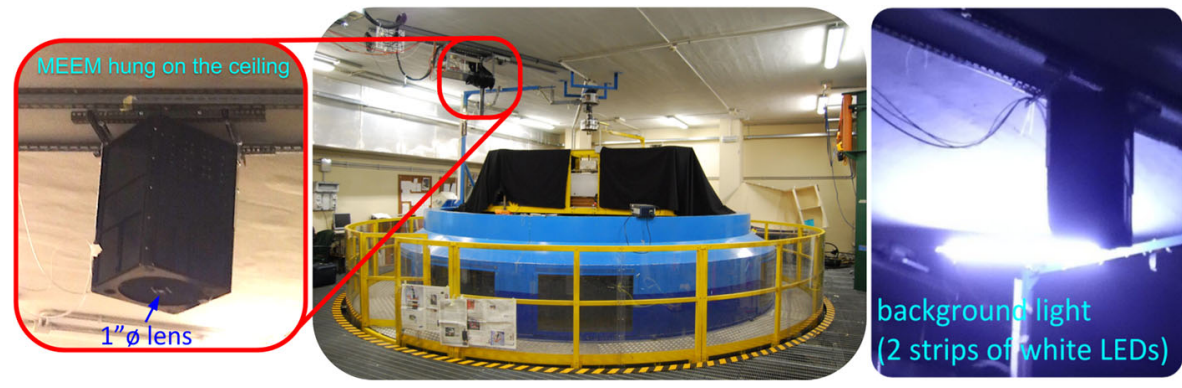

Fig. 3 The TurLab tank setup with the Mini-EUSO EM installed on the ceiling and enlarged inside the red frame (left). The diffused airglow in the atmosphere was reproduced by two strips of cold-white LEDs reflected by the ceiling above the tank (right) 

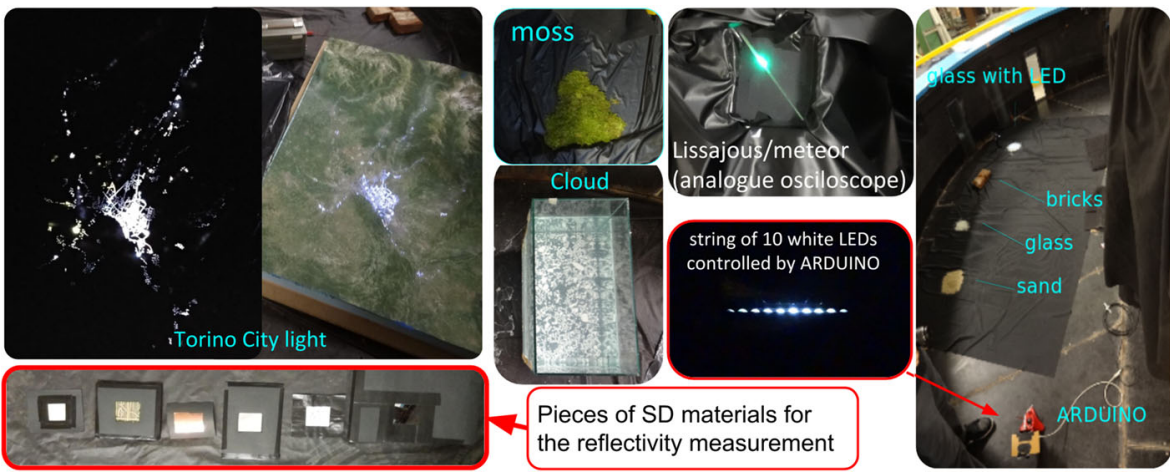

Fig. 4 Light sources and materials to reproduce phenomena that Mini-EUSO can observe. Reflections of materials that are often present in space debris (SD) have also been tested

The setup for the TurLab measurements is shown in Fig. 3. In the central panel the TurLab tank is shown, and on top of it, the detector to be tested is installed on the ceiling as shown in the area surrounded by a red frame in the left panel. The pictures in Fig. 4 show the light sources and materials placed on the floor of the tank to reproduce various phenomena that Mini-EUSO is able to observe from space. In Table 2 materials and phenomena reproduced in the laboratory are listed with the corresponding means of emulation. All materials were illuminated by diffused light, emitted by two strips of cold-white LEDs and reflected by the ceiling above the tank (see Fig. 3 on the right), to reproduce the diffused airglow in the atmosphere at the level of expected photon counts that Mini-EUSO would observe ( $\sim 1$ count/pixel/D1 GTU).

By rotating the tank, it was possible to emulate the Mini-EUSO observation of the Earth from the ISS orbit. In Fig. 5 the light curve measured by Mini-EUSO EM during one TurLab tank rotation is shown, with a time resolution of 1 D3 GTU. In

Table 2 List of materials and phenomena reproduced at TurLab with the corresponding means of emulation

\begin{tabular}{ll}
\hline Materials and phenomena & Means of emulation \\
\hline Rocks & Bricks \\
Deserts & Sand \\
Glacier ice & Smashed glass \\
Clouds & Clusters of particles floating in the water \\
Forests & Moss \\
Lightning & Smashed glass illuminated by blinking LED \\
City lights & LED light through the holes on the map of Turin city \\
Meteors & Lissajous figures generated by an analogue oscilloscope \\
Cosmic rays & Arduino-driven LED strip \\
Space debris & Aluminum foil, copper foil, mirror, white paper, \\
& Kevlar ${ }^{\circledR}$, electronics board \\
\hline
\end{tabular}




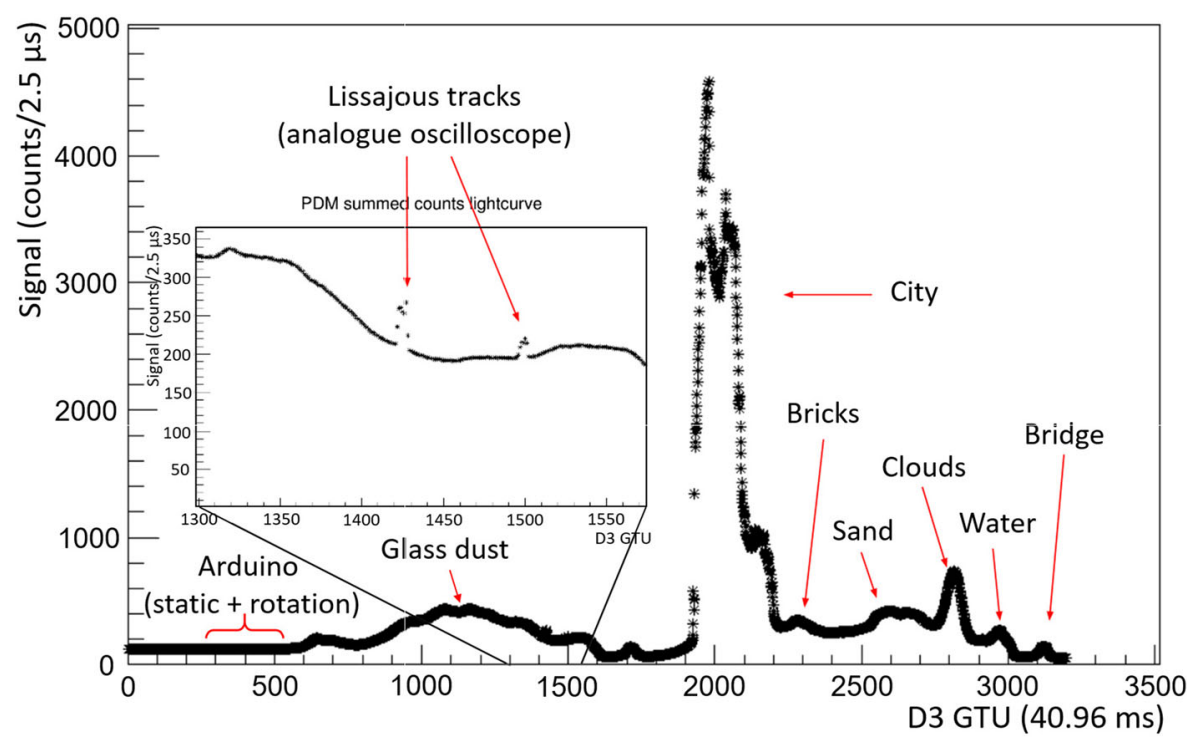

Fig. 5 Light curve of D3 data for a whole tank rotation. All the materials in the field of view are recorded as a continuous "movie". The "Bridge" signature is due to light reflected by a mechanical structure of the tank, partially seen although it was covered by black fabrics to hide it, as not of interest in the context of the measurements. Figure adapted from [16]

the D3 timescale, the light from all the materials in the field of view is recorded as a continuous "movie", so the light curve represents the counts collected in each D3 GTU by the detector while the TurLab tank was rotating below it. The signal is rescaled to $1 \mathrm{D} 1 \mathrm{GTU}(2.5 \mu \mathrm{s})$ as well as in all the plots in this paper.

\subsubsection{Tests on the L1 trigger}

The L1 trigger allows for the detection of cosmic-ray-like events, reproduced at TurLab with a strip of LEDs driven by Arduino. The L1 trigger algorithm looks for signal excess on 8 consecutive D1 GTUs $(20 \mu \mathrm{s})$. For each pixel, the signal integrated over $128 \mathrm{D} 1$ GTUs $(320 \mu \mathrm{s})$ is used to determine the average background level per D1 GTU $\bar{n}$, and therefore the average background counts integrated over 8 consecutive D1 GTUs is $\bar{n}_{8}=\bar{n} \times 8$. The trigger threshold is calculated as $T_{\text {trig }}=\bar{n}_{8}+m \cdot \sqrt{\bar{n}_{8}}$, where the term $\sqrt{\bar{n}_{8}}$ is the standard deviation of the counts integrated over 8 D1 GTUs, assuming they follow a Poisson distribution, and $m$ is an integer number that can be used as parameter to define the trigger performance, initially set to $m=8$. In order to have a trigger, an event has to generate counts integrated over 8 consecutive D1 GTUs greater than the rounded up threshold. In such a case, a packet of 128 D1 GTUs around the triggered event is saved: as 64 D1 GTUs are saved before and after the trigger, the D1 GTU at which the trigger condition is satisfied is the 64th of the packet. The minimum value for the threshold is set to 15 counts, which corresponds to the threshold of a pixel with a background of 0.3 counts/pixel/D1 GTU. Some pixels with low efficiency, and 


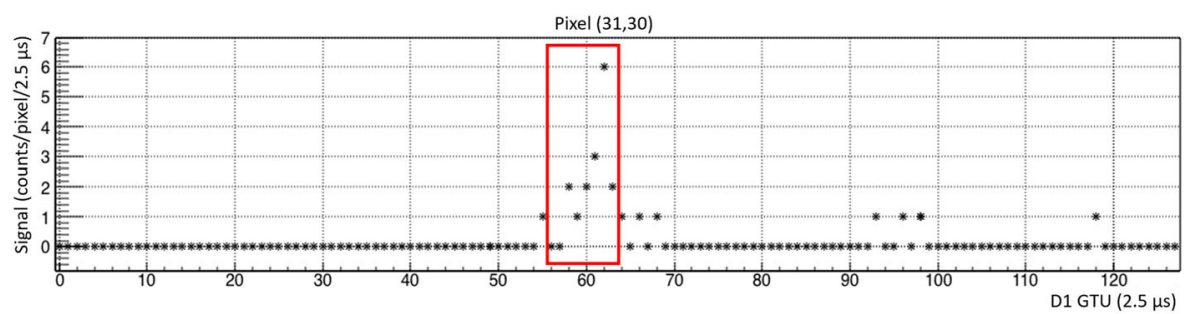

Fig. 6 Light curve over 128 D1 GTUs of a pixel with very low background, $<0.3$ counts/pixel/D1 GTU. The counts integrated over 8 consecutive D1 GTUs exceed the threshold of 15 counts (with 16 counts in the red frame), which is the minimum threshold in very low background condition, and therefore this event was recorded

therefore with low measured background, have been used to test the trigger functionality in case of low background condition, for which the expected threshold was 15 counts. In Fig. 6 the light curve of one of such pixels is shown. Summing up the counts of the last 8 D1 GTUs, at the D1 GTU 63 (the 64th of the packet starting counting from D1 GTU 0) the threshold value is exceeded (16 counts), and therefore this event was recorded. In Fig. 7, the light curve of a pixel with a background of $\sim 0.6$ counts/pixel/D1 GTU is shown. In this case, the threshold is higher than the default minimum one, and it results to be 23 counts. At D1 GTU 63, the sum of the counts over the last 8 D1 GTUs (30 counts) is above the threshold and therefore the event was triggered.

Tests performed with the Mini-EUSO EM at TurLab showed that the average trigger rate was around $10 \mathrm{~Hz}$, much higher than the required limit of $0.75 \mathrm{~Hz}$. The high trigger rate was due to the low efficiency pixels mentioned before, which do not follow the Poissonian behavior due to the electric noise or to intrinsic characteristics of the MAPMT. The signal measured by such pixels easily exceeded the minimum threshold, inducing an unexpected high trigger rate. For this reason, subsequent tests using a system similar to the Mini-EUSO EM have been performed. These tests indicated that to keep the trigger rate below $0.75 \mathrm{~Hz}$ at all background levels (from $\leq 0.3$ counts/pixel/D1 GTU to city lights), the $m$ parameter in the formula to calculate the threshold had to be fixed to $m=16$.

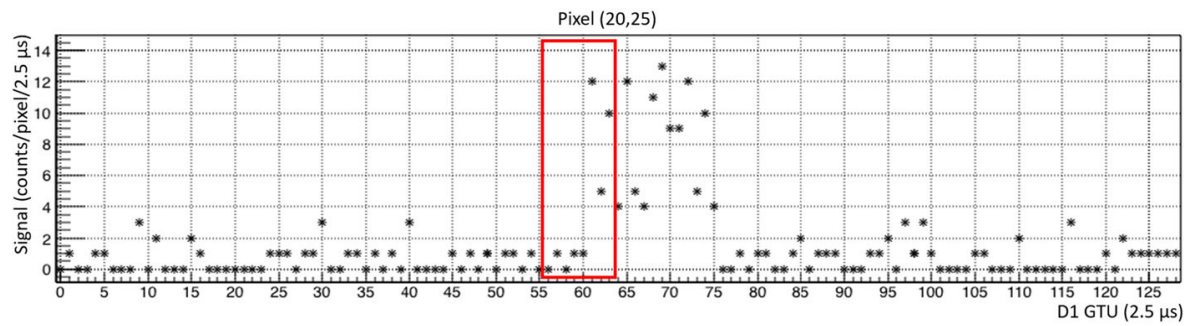

Fig. 7 Light curve over 128 D1 GTUs of a pixel with a background of $\sim 0.6$ counts/pixel/D1 GTU. The counts integrated over 8 consecutive D1 GTUs reach the threshold of 23 counts (with 30 counts in the red frame), and therefore this event was recorded 


\subsubsection{Tests on the L2 trigger and D3 continuous data acquisition}

At TurLab, also the L2 trigger was tested. Bright events lasting relatively long such as Lissajous figures produced by an analogue oscilloscope, which can give similar time profiles to those of transient luminous events, were detected using the L2 trigger. It also triggered on the mimicked city light, as the light increased rapidly when it entered the field of view of the detector from inside the tank, but any other material or light source such as glass dust or the Arduino-driven LED strip did not generate any trigger, i.e., no fake triggers occurred during this test.

Also, as shown in Fig. 5 for the D3 data, a continuous data acquisition was stored during the measurements, and each illuminated material and light source can be clearly recognized in the light curve. In Fig. 8 samples of observations made in the D3 timescale are shown. The panel (a) shows the signal from Lissajous figures of an analogue oscilloscope reproducing a meteor, integrated over 10 D3 GTUs in order to see the whole track in one frame. The panels (b) and (c) show smashed glass reproducing glacier ice and sand reproducing desert, respectively.

\subsubsection{Measurements for space debris studies}

Space debris do not emit light themselves but can be detected if illuminated by sunlight. Measurements of the reflectivity of materials which usually compose space debris (shown in the bottom-left of Fig. 4) were performed at TurLab [17]. For these tests, the Mini-EUSO EM was hung on the ceiling above the tank pointing downward. Different materials such as polished and unpolished aluminum foil, canned aluminum foil, balled aluminum foil, copper foil, mirror, white paper, a sample of $\operatorname{Kevlar}^{\circledR}$, and electronics board (all materials that often space debris are composed of), were placed on the bottom of the tank and illuminated by diffused light with different intensities. In this way, the relative reflectance of the materials as seen by the detector was measured. As a preliminary estimation, assuming that the reflec-

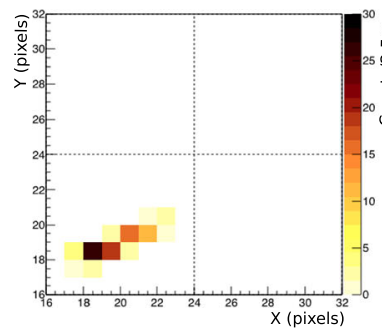

(a)

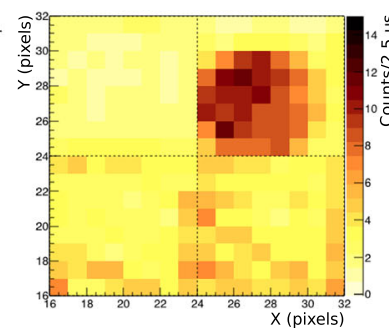

(b)

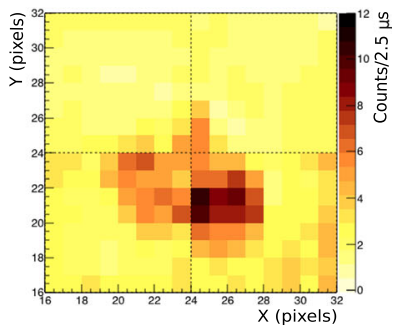

(c)

Fig. 8 Examples of D3 frames for: (a) Lissajous figures of an analogue oscilloscope (meteor), (b) smashed glass (glacier ice), and c sand (desert). In panel (a) the signal from Lissajous figures is integrated over 10 D3 GTUs to visualize the moving track in one data frame. Panels (b) and (c) refer to single D3 frames and include background 
tivity of polished aluminum is about 0.92 , the reflectance of the different materials varying from 0.17 (electronics board) to 0.92 (fixed reference value for the polished aluminum foil) was measured.

Simulation studies applying a dedicated trigger algorithm indicated the capability of a Mini-EUSO-like detector to observe and track space debris. Such detectors need a shade to prevent direct sunlight reaching the lens without hindering the field of view, otherwise stray light brings protection-mode to not damage the device. By using ESAF (EUSO Simulation and Analysis Framework) [18] an estimation of the minimum sizes and maximum distances of detectable space debris was made [17]. Furthermore, the number of expected space debris detections was estimated by using an ESA software called MASTER (Meteoroid and Space debris Terrestrial Environment Reference) [19], which provides the density of space debris with parameters such as altitude and inclination. Such a detector was evaluated to have a potential detection rate of hundreds of space debris per year [17]. An important contribution was provided by the detection of a real space debris with the Mini-EUSO EM during a data acquisition session at the Astrophysical Observatory, discussed in Section 4.3.2. Size and distance of this rocket body have been rescaled in order to reproduce the detection of space debris with Mini-EUSOlike detectors. These studies are important to verify the working principle of a space-based system under development for the detection and remediation of space debris [5].

\section{Measurements in open sky conditions}

\subsection{Mini-EUSO EM at the Astrophysical Observatory of Turin}

The Astrophysical Observatory of Turin, located on a hill at Pino Torinese, about $7 \mathrm{~km}$ east of Turin, offers good conditions for the observation of the sky, as city lights are relatively far away and at lower altitude with respect to the observatory site. Moreover, it hosts one all-sky camera of the Italian network for systematic surveillance of meteors and atmosphere, PRISMA [20].

The PRISMA network is devoted to the observation of fireballs in the sky, with the goal of recovering the meteorites surviving the ablation in the atmosphere. On 1 January 2020 at 18:26:53 UTC, eight cameras of the network observed a bolide that allowed precise estimation of the area where to search for fragments (strewn-field). On 4 January, the meteorite was recovered in the estimated area. This was the first meteorite recovered thanks to a fireball network in Italy, and the 20th in the world so far [21].

The PRISMA cameras observe the sky with a wide wavelength bandwidth peaked in the visible band and with an angular resolution of $\sim 0.2^{\circ} \times 0.2^{\circ} /$ pixel [22]. They operate with a high frequency of $30 \mathrm{~Hz}$ (with $1 / 30 \mathrm{~s}$ exposure time) to follow the development of fireballs properly. Slow events such as planes or satellites are not recorded, as they are rejected by the trigger algorithm specifically designed for meteor detection. At this exposure time, stars are not recognizable. Therefore, 
additional ancillary images integrated over $5 \mathrm{~s}$ are saved every 10 minutes, in order to use star images to derive astrometric and photometric calibration of the instrument [23]. In Fig. 9 one of such all-sky images for the observation of stars is shown. On the edge of the image, bright spots correspond to the nearby urban areas.

A comparison of the stars observation with the PRISMA camera and the MiniEUSO EM was made to understand the relative performance of the two detectors, also in view of possible future simultaneous observations of meteors with the PRISMA cameras from the ground and Mini-EUSO from the ISS. A comparison of the rising signal at dawn time was also performed. In addition, from the same site faint meteors and the rocket body of a telecommunication satellite were observed by the MiniEUSO EM, but not by the PRISMA camera.

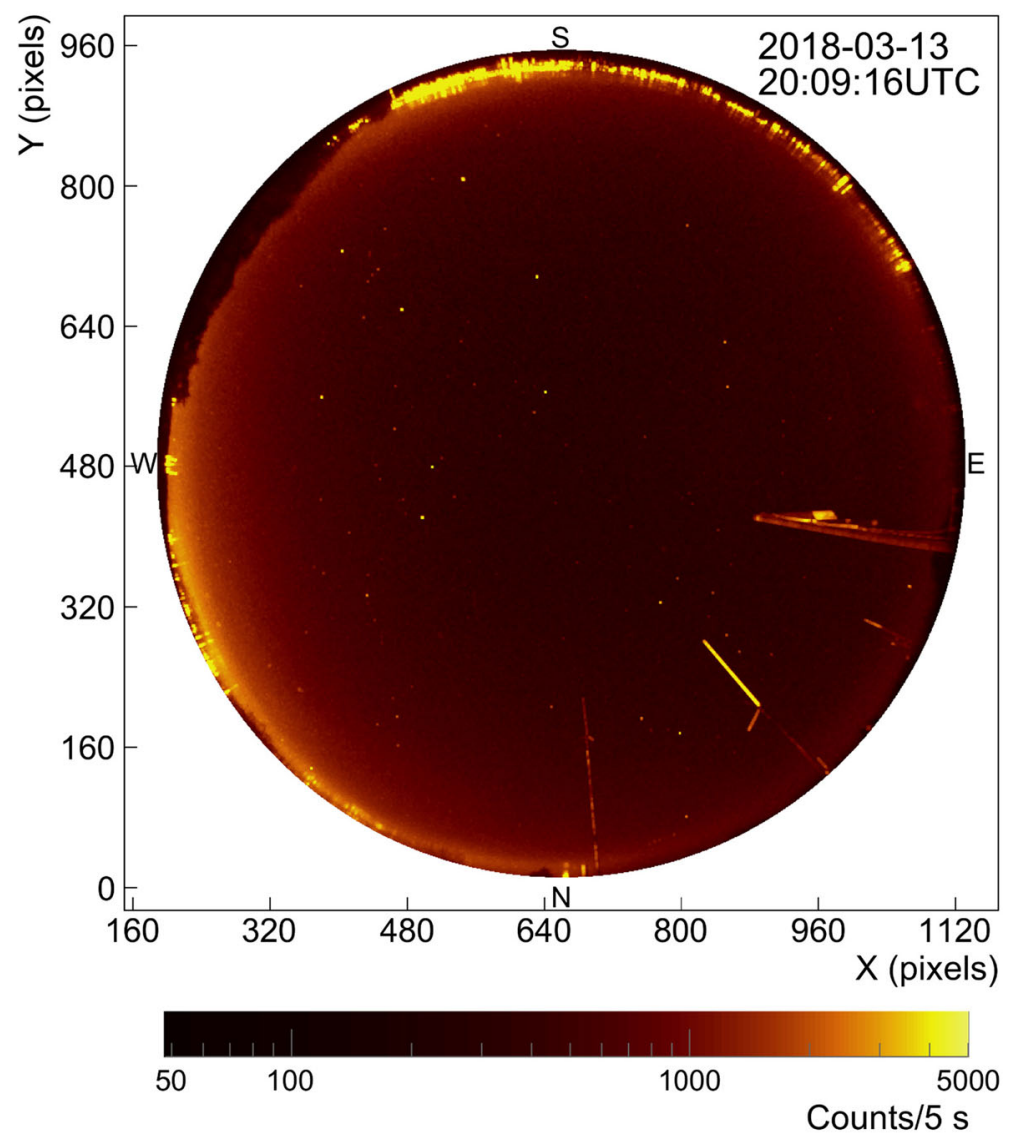

Fig. 9 An image taken by the PRISMA camera during the Mini-EUSO EM data acquisition campaign of 13 March 2018. The image is integrated over $5 \mathrm{~s}$ for the observation of stars. Near the horizon, bright spots correspond to the nearby urban areas. A flag and antennas present at the PRISMA camera site are seen on the north-east part of the field of view. The scale of this image is modified from the output values to visualize brightnesses of both the night sky and stars 


\subsection{Natural sources}

\subsubsection{Stars and planets}

Several stars and the planet Jupiter have been identified in the data taken with the Mini-EUSO EM from the Astrophysical Observatory by pointing at different zenith angles during the nights of the data acquisition. By comparing the cataloged positions of the stars with the observations, the total field of view of the instrument was estimated as $\sim 10^{\circ} \times 10^{\circ}$, or $\sim 0.6^{\circ} \times 0.6^{\circ} /$ pixel.

The simultaneous observations of stars, i.e., with the same atmospheric condition, allow to compare the observations made with the two detectors. In Fig. 10 the panel (a) shows the count rates from Jupiter and the stars observed by the Mini-EUSO EM (red y-axis on the left) and by the PRISMA camera (blue y-axis on the right). The count rates are shown as a function of the observed U-band and V-band apparent magnitudes, respectively, with the atmospheric attenuation correction depending on the zenith angle of the stars. In the panels (b) and (c) some stars of the Orion constellation are shown in a data frame of the Mini-EUSO EM, integrated over $40.96 \mathrm{~ms}$ (1 D3 GTU), and one of the PRISMA camera, integrated over $5 \mathrm{~s}$, respectively. The former has been mirrored to have the same orientation of the latter. Jupiter, Sirius and the stars of the Orion constellation were detected at zenith angles $>60^{\circ}$, while Alkaid and the Hercules' stars were detected close to the zenith. In general the count rates in logarithmic scale decrease linearly with the apparent magnitude, particularly for the stars at the zenith. Taking as example the star $\alpha$ Ori (apparent magnitudes in the catalog: $\mathrm{U}=+4.38, \mathrm{~V}=+0.42$ ), the observed magnitudes in the $\mathrm{U}$-band and $\mathrm{V}$ band have very different values, as it is a red-giant star of the Orion constellation. The corresponding measured counts are consistent with the expectations. Very bright and very dim stars represent some outliers. Jupiter and Sirius seen by PRISMA present fewer counts than what is expected from the linear trend due to saturation of the sensor. 30 Her $(\mathrm{U}=+7.70, \mathrm{~V}=+5.01)$ was not detected by the Mini-EUSO EM because of its low emission in the U-band, while it is brighter than expected from the observed V-magnitude. 32 Ori $(\mathrm{U}=+3.51, \mathrm{~V}=+4.2)$ was invisible to PRISMA at the time of the Mini-EUSO EM observation due to its high zenith angle and therefore its high observed $\mathrm{V}$-magnitude due to the atmospheric absorption. $v$ Her $(\mathrm{U}=+4.33$, $\mathrm{V}=+4.71$ ) was not recognizable in the PRISMA frame even if the star was close to the zenith, because of its sensitivity limit. Indeed, as PRISMA is optimized for the detection of bright meteors, the limit for the apparent magnitude for the $5 \mathrm{~s}$ integration frames is about +4.5 . In both the Mini-EUSO EM and the PRISMA camera the stellar signal can be shared by adjacent pixels, and as a consequence the signal per pixel can be reduced (or partially lost in the case of the Mini-EUSO EM, where the pixels are less sensitive close to their edges) and stars might not be distinguishable from the night sky background, particularly for dim stars. Moreover, in the analysis the U-band and the V-band apparent magnitudes were considered for the comparison, but both the devices are sensitive to a wider range of wavelengths than the bandwidths for which the apparent magnitudes are defined. In general, and limited to the 


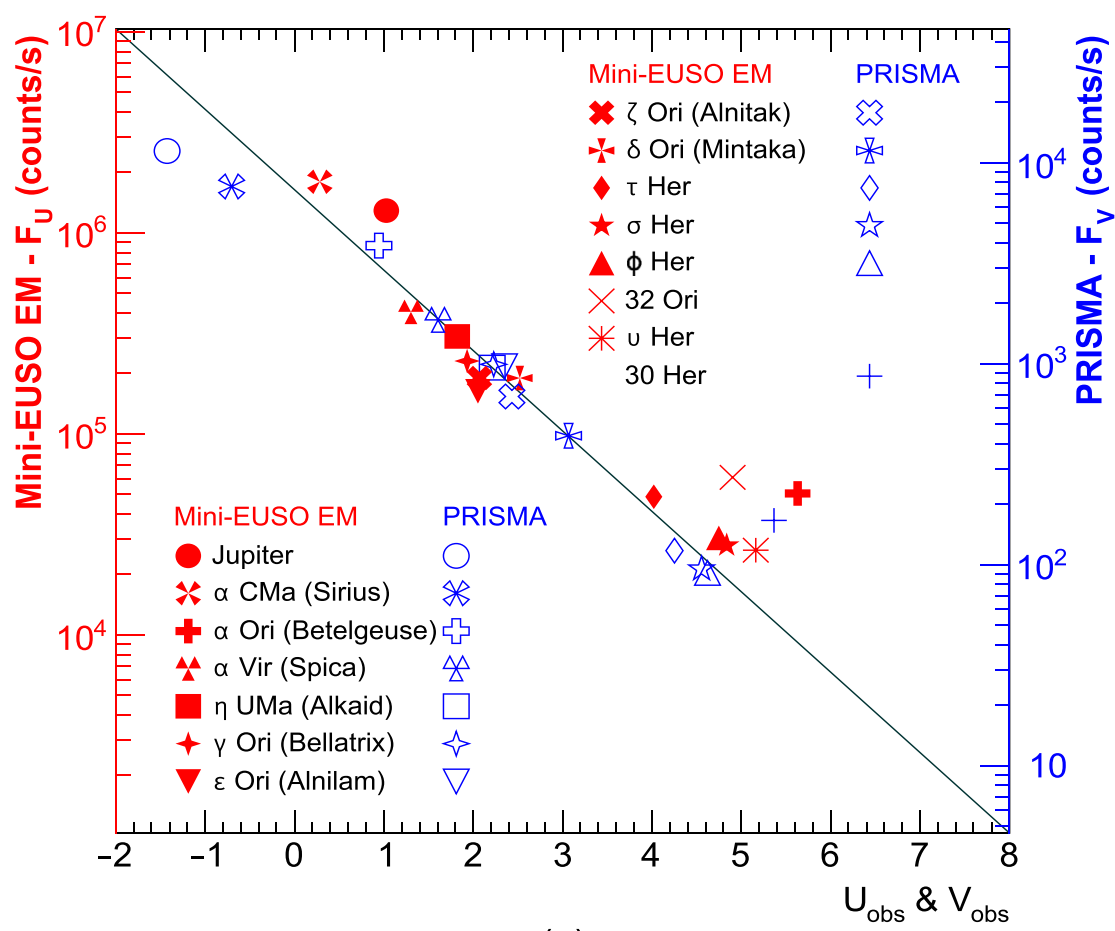

(a)

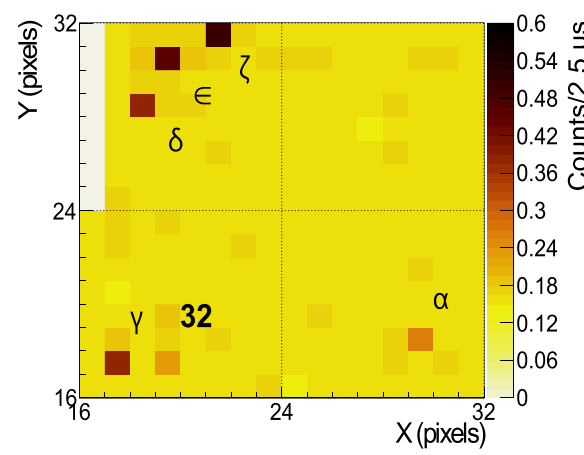

(b)

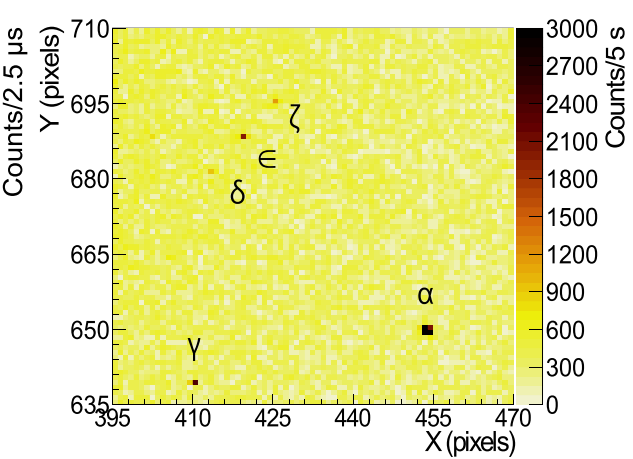

(c)

Fig. 10 (a) Count rates from stars and Jupiter for the Mini-EUSO EM and for the PRISMA camera, with respect to the observed $\mathrm{U}$ and $\mathrm{V}$ apparent magnitudes, respectively. (b) Some of the stars of the Orion constellation in a D3 frame of the Mini-EUSO EM integrated over $40.96 \mathrm{~ms}$ and (c) the same stars in a frame of the PRISMA camera integrated over $5 \mathrm{~s}$, where the 32-Ori star is not recognizable. Image (b) has been mirrored to have the same orientation of image (c)

sample of observed stars, the Mini-EUSO EM was sensitive to apparent magnitudes from astronomical catalog up to about +4.4 and observed apparent magnitudes up to about +5.6 . The limiting magnitude would increase using exposures longer than 1 D3 GTU. 


\subsubsection{Meteors}

The analysis of stars measured by both the Mini-EUSO EM and the PRISMA camera was useful in the case of simultaneous observation of meteors by Mini-EUSO from space and by the PRISMA cameras or other meteor observatories on ground that might operate in a similar manner. Indeed, usually meteors are observed in the visible band, and the observation in the UV band gives additional information about the development of meteors in the atmosphere. Moreover, observations from very different viewing angles during their development could improve the understanding of these phenomena. More details on the observation of meteors with Mini-EUSO are available in [24].

Four meteor candidate events have been detected while the Mini-EUSO EM was pointing in the zenith direction at the Astrophysical Observatory. From the comparison with the brightness of stars, they had apparent magnitudes of about +4 . There is no counterpart of these events in the data of the PRISMA camera, as it is not sensitive to such faint meteors. Indeed, its limit for the apparent magnitude is about -1 , or even about 0 with the best sky conditions. As the meteor candidates developed near the zenith, the horizontal component of their speed was estimated by supposing that the development of meteors starts at $\sim 100 \mathrm{~km}$ altitude. It was estimated as tens of kilometers per second, consistent with the typical meteor speeds.

In Fig. 11 one of the meteor candidates is shown, moving through four consecutive D3 frames in the top MAPMTs along 8 pixels, from right to left. The duration of this event was $\sim 160 \mathrm{~ms}(\sim 20 \mathrm{~ms} /$ pixel $)$ and the estimated horizontal speed was $\sim 50 \mathrm{~km} / \mathrm{s}$.

\subsubsection{Night sky brightness}

A measurement of the night sky brightness was performed over until dawn on 14 March 2018. The increase of the brightness was clearly detected by both the MiniEUSO EM, while it was pointing to the zenith, and the PRISMA camera. In Fig. 12 the count rates of the four MAPMTs of the Mini-EUSO EM are shown. The background brightness obtained from the PRISMA data with an aperture of $15^{\circ}$ around the zenith is compared by normalizing $19 \mathrm{mag} / \mathrm{arcsec}^{2}$ to 0.05 counts/pixel/2.5 $\mu$ s of the Mini-EUSO EM.

At the Astrophysical Observatory, the night sky is polluted by the urban light in the Turin area. This brightness is consistent with the zenith sky brightness of $19.3 \mathrm{mag} / \mathrm{arcsec}^{2}$ provided in [25] that showed the contribution of artificial light sources being $\sim 11$ times of the intrinsic sky brightness. The corresponding values in the middle of the ocean are greater than $22 \mathrm{mag} / \mathrm{arcsec}^{2}$, namely more than 10 times darker than that observed by this work. In general, the areas dominated by artificial light sources are not suitable for the observation of EASs from space and the range of the observable energy of UHECRs is limited to be high. The fraction of such areas below the ISS was estimated to $\sim 10 \%$ [2] based on the DMSP satellite data [26]. 


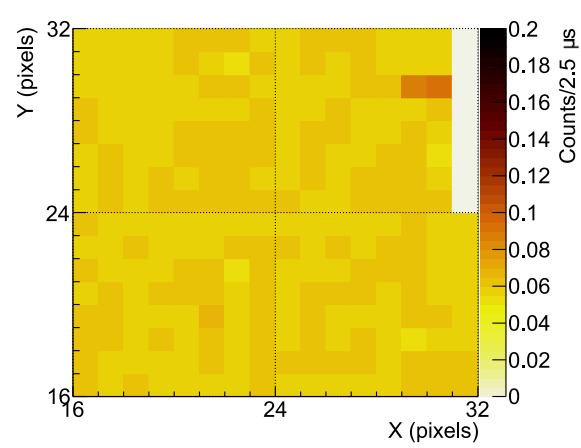

(a)

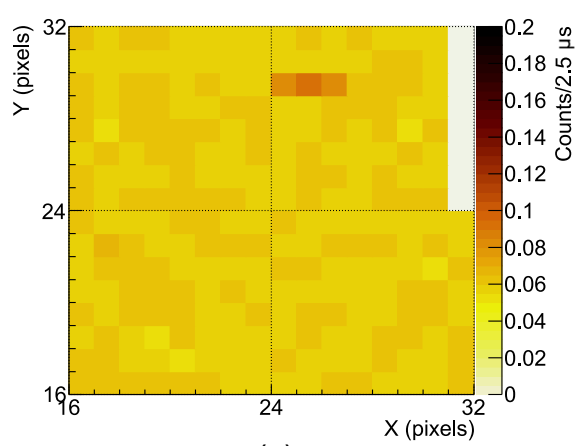

(c)

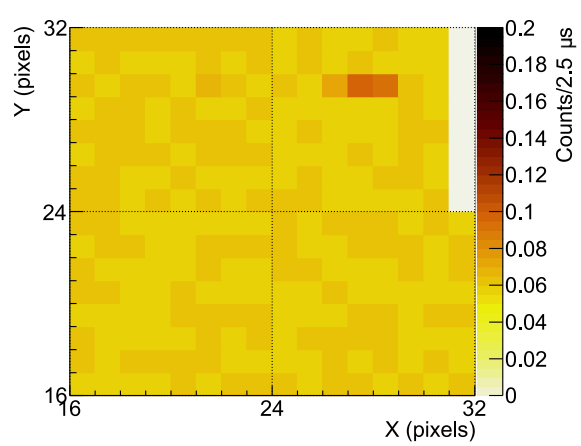

(b)

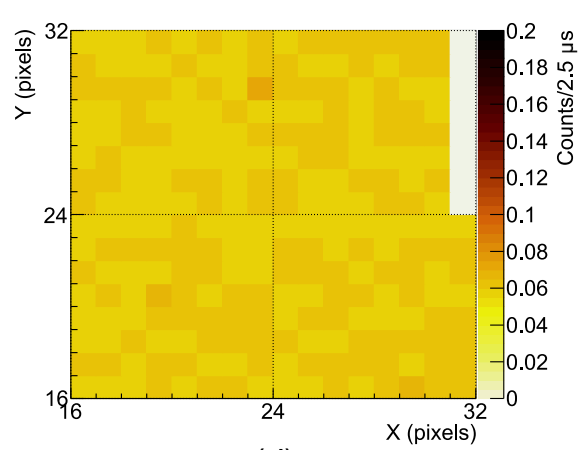

(d)

Fig. 11 Example of a meteor candidate shown in four consecutive D3 frames, each integrated over $40.96 \mathrm{~ms}$. Figure adapted from [27]

The astronomical twilight started at 04:05 UTC prior to the sunrise at 05:45 UTC. The count rate of Mini-EUSO EM in the UV band increased first, followed by the count rate of the PRISMA camera in the visible band. This can be explained by taking into account that UV photons in the atmosphere are more scattered than the visible ones. Therefore, with the Sun approaching the horizon before sunrise, a higher flux of scattered light is observed at the zenith in the UV band rather than in the visible band. The Moon with $11 \%$ illumination rose above the horizon at 04:20 UTC, but its effect on the scattered light near the zenith was marginal.

On the light curve of MAPMT 3, higher count rates are seen between 01:31 and 02:02 UTC when the star Alkaid, with the U-band apparent magnitude of about +1 , crossed the field of view of this MAPMT over an angle of $\sim 5^{\circ}$, equivalent to $\sim 9$ pixels length. This corresponds to a $\sim 0.2^{\circ} / \mathrm{min}$ angular speed, which is consistent with the diurnal motion for stars culminating near the zenith at the latitude of the Astrophysical Observatory. In the count rate due to Alkaid, peaks correspond to the star signal hitting the center of the pixels and the valleys correspond to the edges between adjacent pixels, where the sensors are less sensitive. 


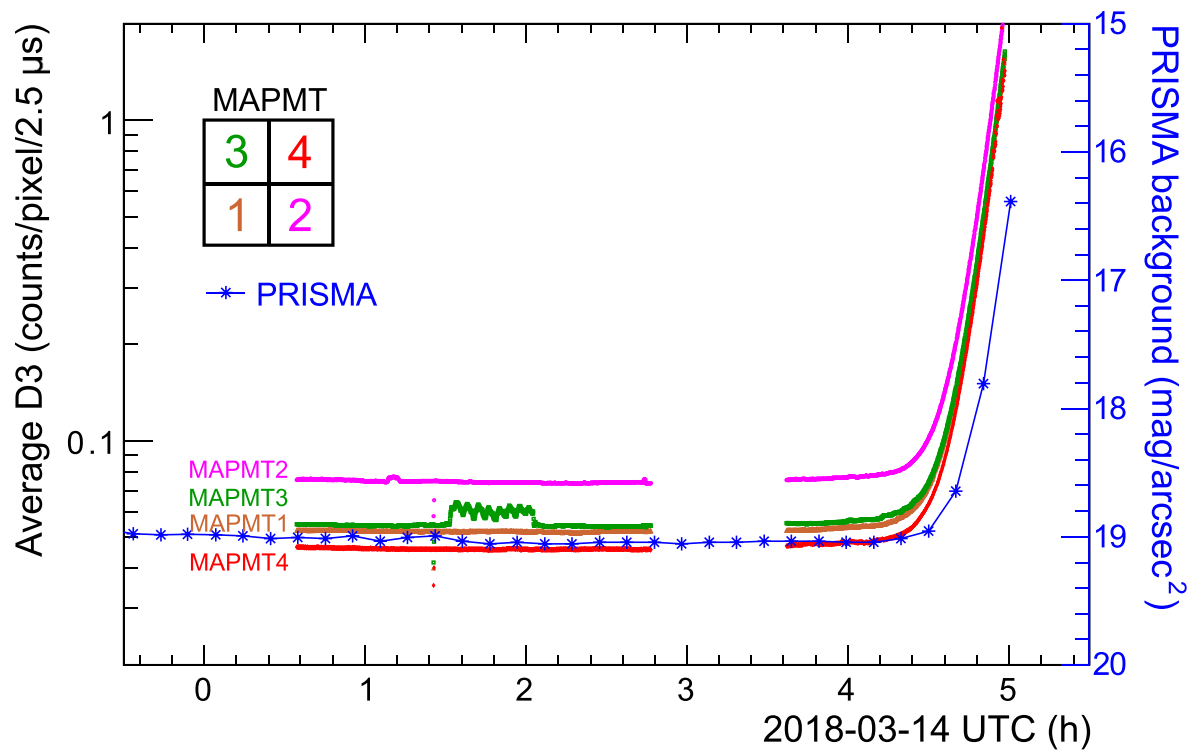

Fig. 12 Night sky count rate over time of the four MAPMTs of the Mini-EUSO EM (scale on the left) and of the PRISMA camera (scale on the right). The measurement made with the PRISMA camera is normalized at $19 \mathrm{mag} / \mathrm{arcsec}^{2}$ to 0.05 counts/pixel/2.5 $\mu$ s of the Mini-EUSO EM

\subsection{Artificial sources}

\subsubsection{Lit signs, flashes and city light}

Artificial lights from the nearby urban area were also used to test the detector. From the roof of the Physics Department, lit signs and warning lights on skyscrapers and towers located in the Turin area (at 2-4 km distance) were detected, as well as lights from Trofarello and Chieri towns from the Astrophysical Observatory.

In Fig. 13 a picture of the "Mole Antonelliana" tower is shown in the panel (a), together with the light curve of one pixel with time bins of $320 \mu \mathrm{s}$ (D2 timescale) with the signal from the tower in the panel (b). The light curve shows an alternating signal with a period of $\sim 35$ time bins, corresponding to a frequency of $\sim 100 \mathrm{~Hz}$. The frequency of the electrical grid is $50 \mathrm{~Hz}$ in Italy. Artificial light sources connected to an alternating current fluctuate in different manners depending on the lighting technology. Incandescent bulbs fluctuate at the frequency of the electrical supply $(50 \mathrm{~Hz})$, while fluorescent lighting fluctuate twice over each voltage cycle $(100 \mathrm{~Hz})$. Therefore, the measured modulation is consistent with the frequency of fluorescent lighting connected to the electrical grid. In the panel (c) a picture of the "Intesa Sanpaolo" skyscraper with constant emission and white/blue blinking warning lights is shown, together with light curves on two timescales. The light curve with time bins of $320 \mu \mathrm{s}$ in the panel (d) shows an alternating trend with a frequency of $\sim 100 \mathrm{~Hz}$, like in the panel (b). The light curve with time bins of $40.96 \mathrm{~ms}$ in the panel (e) shows high peaks due to flashes that are well above the baseline, pulsating with a frequency 


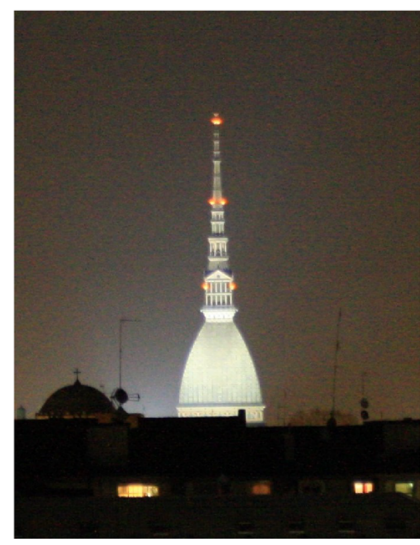

(a)

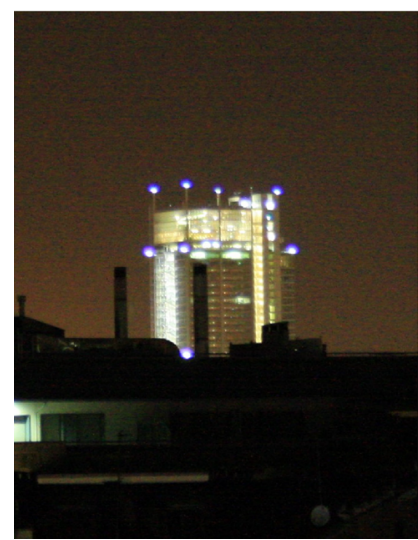

(c)

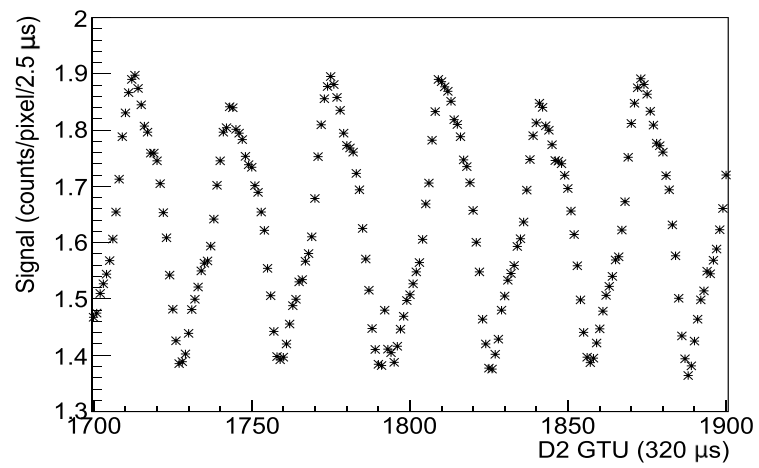

(b)

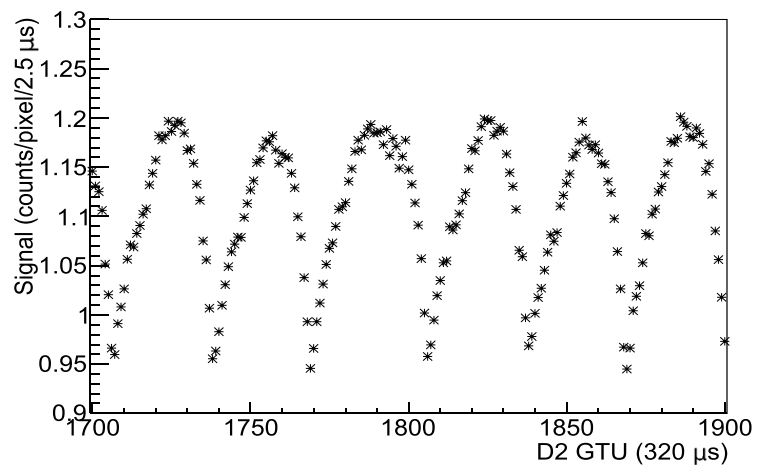

(d)

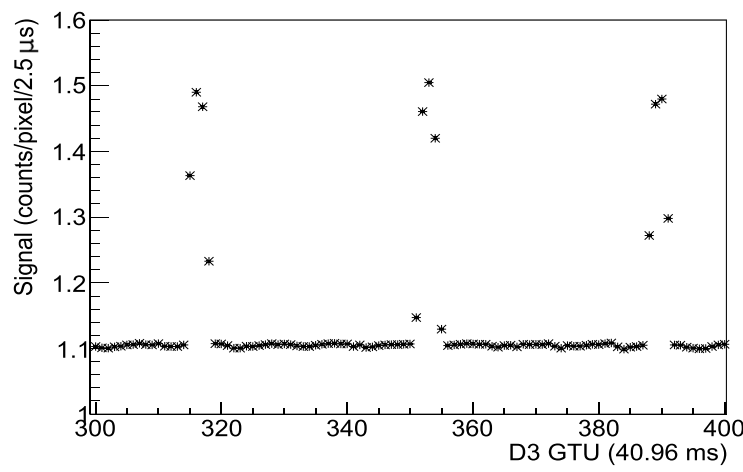

(e)

Fig. 13 (a) Picture of the "Mole Antonelliana" tower; (b) light curve with the signal from the tower, with time bins of $320 \mu \mathrm{s}$ (D2 GTUs), showing the alternating signal with frequency of $\sim 100 \mathrm{~Hz}$. (c) Picture of the "Intesa Sanpaolo" skyscraper; (d) light curve with the signal from the skyscraper as same as panel (b), and (e) with time bins of $40.96 \mathrm{~ms}$ (D3 GTUs), showing flashes from the warning lights with frequency of $\sim 0.7 \mathrm{~Hz}$. The signals with a frequency of $\sim 100 \mathrm{~Hz}$ are consistent with the $50 \mathrm{~Hz}$ frequency of the electrical grid. The measurements pointing the detector to the "Mole Antonelliana" tower and the "Intesa Sanpaolo" skyscraper were made at different times. Figure adapted from [27] 
of $\sim 0.7 \mathrm{~Hz}$. The $\sim 100 \mathrm{~Hz}$ frequency pattern is present on the baseline and on the peaks as well. Other buildings with flashers have similar pulsating light curves, and also generic light from cities have light curves with such an alternating profile.

The alternating pattern in light curves for the D2 timescale is an important feature of the signal that allows to discriminate between natural and anthropogenic sources. Indeed, with the first observations of the Earth with Mini-EUSO from space, the modulation of the light current of $50 \mathrm{~Hz}$ or $60 \mathrm{~Hz}$ was recognized, depending on the observed area of the globe. It also detected flashes. These observations are described in more details in Section 5.

\subsubsection{Rocket of a telecommunication satellite and airplane}

During the observations at the Astrophysical Observatory, while pointing to the zenith, the orbiting rocket body "Meteor 1-31 Rocket" (NORAD-ID 12586) was detected on 14 March 2018 at 04:10:58 UTC. This device was used for the launch of a telecommunication satellite in 1981 and was left in orbit as a $2.6 \mathrm{~m} \times 2.8 \mathrm{~m}$ largesize debris orbiting at an average height of $\sim 550 \mathrm{~km}$ and a velocity of $\sim 7.5 \mathrm{~km} / \mathrm{s}$. The satellite was identified with the Systems Tool Kit (STK) platform [28]. The signal from the satellite is shown in Fig. 14 as a bright pixel moving from top-left to bottom-right in the EC, in four D3 frames integrated over $40.96 \mathrm{~ms}$ and sampled every $\sim 4$ s. The event lasted for 400 D3 GTUs $(\sim 16 \mathrm{~s})$. The satellite crossed a length equivalent to $\sim 22$ pixels in the inclined direction, including also the gaps between the MAPMTs $(\sim 1.4$ pixels/s). By considering these parameters, the field of view of one pixel of the Mini-EUSO EM, and the average height of the rocket body, a speed of $\sim 7.7 \mathrm{~km} / \mathrm{s}$ was estimated, consistent with the cataloged one.

This measurement can be translated to an equivalent observation performed by a Mini-EUSO-like detector from the ISS. In the case such a detector, with the field of view of one pixel of $\sim 0.8^{\circ} \times 0.8^{\circ}$, would observe an event with a speed of $\sim 1.4$ pixels/s, the observation could correspond to a space debris with an apparent speed of $\sim 1 \mathrm{~km} / \mathrm{s}$ at the distance of $50 \mathrm{~km}$. The apparent speed should be corrected by the ISS speed. More detailed simulation studies and rescaling of the size and distance of this satellite have been done in order to reproduce the detection of space debris with a Mini-EUSO-like detector on board the ISS [17].

In addition, flashes of the airplane operating the flight LH1902 from Munich to Turin was detected, from the same site, on 12 March 2018 at around 21:5421:55 UTC (about 6 minutes before landing). In such time interval, it passed $\sim 4-6 \mathrm{~km}$ away at an altitude of $\sim 800-700 \mathrm{~m}$ above the observation site at $610 \mathrm{~m}$ a.s.l., with a speed of $\sim 430 \mathrm{~km} / \mathrm{h}$. The flight was identified via the Flightradar 24 tracking service [29] that provides real-time information about aircrafts around the world. The airplane observation is detailed in Fig. 15 as the average count rates of four MAPMTs on the top and that of the whole EC taking into account the different efficiencies of the MAPMTs, according to rates measured on 14 March at $~ 02: 30 \mathrm{UTC}$, as in Fig. 12. The frequency of the apparent blinking flashes recorded by the EC changed over the time from $\sim 1.3$ to $\sim 2.6$ flashes per second due to the turning of the airplane while approaching the runway. The Mini-EUSO EM was manually pointed towards 


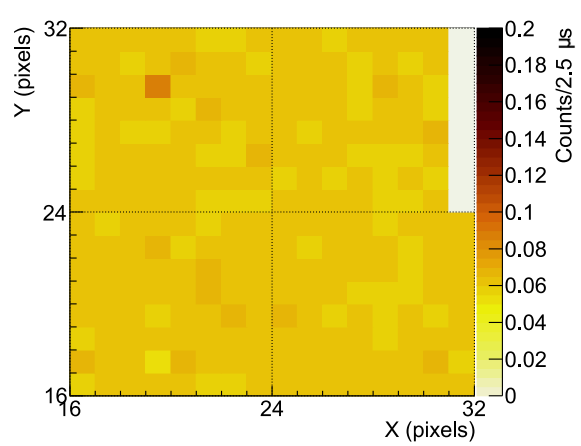

(a)

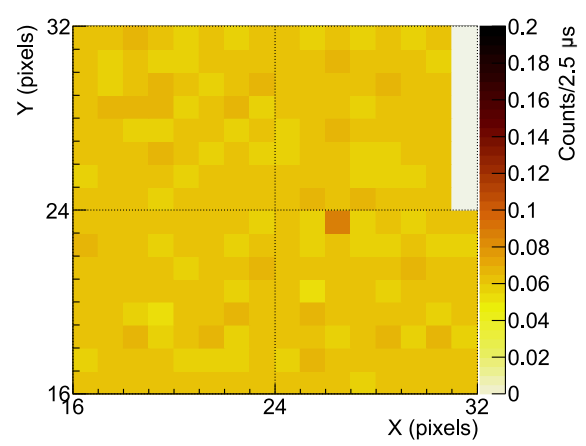

(c)

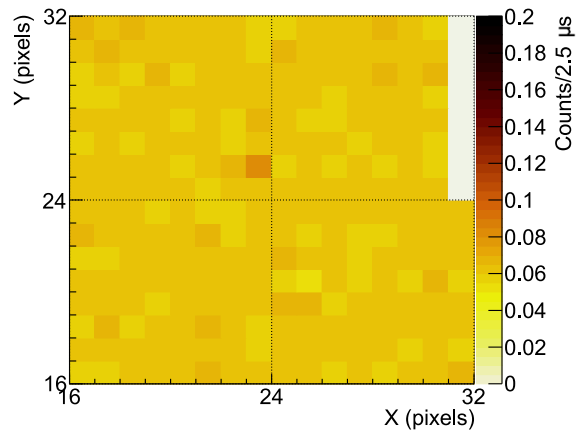

(b)

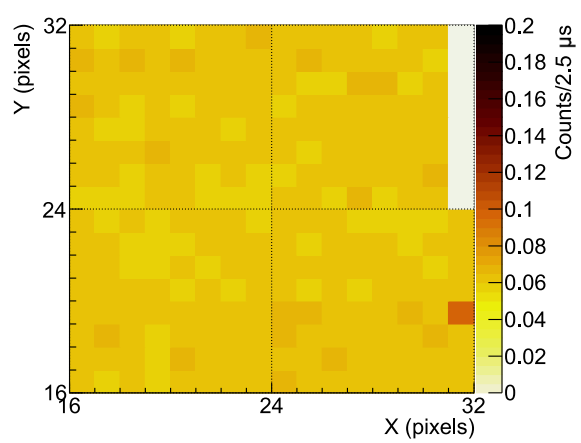

(d)

Fig. 14 Signal from the rocket "Meteor 1-31 Rocket" in four D3 frames integrated over 40.96 ms and sampled every $\sim 4 \mathrm{~s}$. The rocket is recognizable as a bright pixel crossing the field of view from top-left to bottom-right

the airplane. Thus, transition of significant signals among MAPMTs does not represent the motion of the airplane. All MAPMTs show synchronous increased count rates due to the airplane flashers. This is considered due to the light from the flasher scattered in the hazy sky.

\section{Comparing Mini-EUSO EM tests with preliminary Mini-EUSO data}

As discussed in Section 4.3.1, data acquisitions of artificial light from lit buildings and warning flashers with the Mini-EUSO EM demonstrated the capability of this type of system to detect flashes with frequency $\sim 0.7 \mathrm{~Hz}$ (seen in the D3 data, i.e., with frames integrated over $40.96 \mathrm{~ms}$ ) and even the modulation of the alternating current, which is $50 \mathrm{~Hz}$ in Italy (seen in the D2 data, i.e., with frames integrated over $320 \mu \mathrm{s}$ ) - see Fig. 13. Mini-EUSO from space detected the electrical grid modulation of $50 \mathrm{~Hz}$ or $60 \mathrm{~Hz}$, depending on the observed area of the globe. Such a modulation is clearer in small towns and villages that are all connected to the same transformer and therefore emit light in phase. However, it is more difficult to see the modulation 


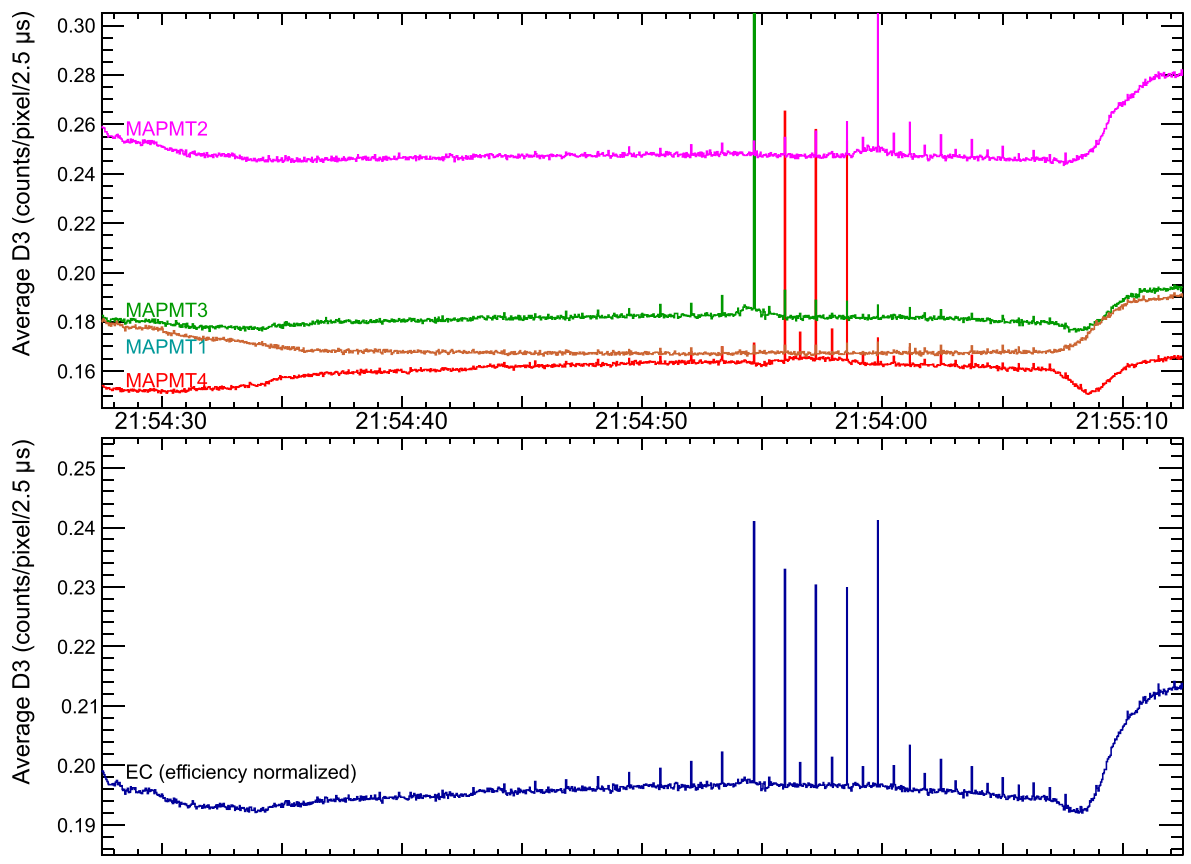

Fig. 15 Light curve of the airplane of the flight LH1902 detected on 12 March 2018 at around 21:5421:55 UTC. In the top panel, average count rates of four separate MAPMTs are shown. In the bottom panel, the average of the whole EC is shown, taking into account different efficiencies of the MAPMTs

in larger cities, where different areas are connected to different transformers, varying phase. Mini-EUSO also observed flashes from several places on the Earth. Their origin is still under investigation, but they seem to be compatible with warning lights mounted on buildings or towers (usually with xenon, with portion of the emission spectrum in the near-UV band). They can have different brightness and duration [6], usually lasting a few hundred microseconds and being observed several times as they cross the field of view of the detector.

Meteors were also observed by both detectors. The observation of meteors made with the Mini-EUSO EM was discussed in Section 4.2.2. A definitive confirmation that the observed objects were meteors cannot be obtained because there was no counterpart in the PRISMA camera data, being too faint for the PRISMA detectability. However, their estimated horizontal speed was consistent with the typical speeds of meteors. Moreover, the spatial and temporal development of the meteors observed by Mini-EUSO in orbit, after the necessary rescaling for the distance between detector and source, seem to be compatible with the properties of the objects observed from the ground by the Mini-EUSO EM. However, while the apparent magnitude of the meteors observed with the prototype could be estimated thanks to the comparison with the U-band apparent magnitude of field stars, several methods are under study for the estimation of the apparent magnitude of meteors detected by Mini-EUSO from the ISS. Taking as an example the meteor in Fig. 11, it is possible to evaluate how Mini-EUSO would have detected the same event from the ISS. Assuming 
that meteors start their development at $100 \mathrm{~km}$ altitude, the area observed by a pixel of the Mini-EUSO EM at this altitude is $\sim 1.0 \mathrm{~km} \times 1.0 \mathrm{~km}$. A pixel of Mini-EUSO on the ISS would observe an area of $4.2 \mathrm{~km} \times 4.2 \mathrm{~km}$ at the same altitude $(300 \mathrm{~km}$ below it). As the meteor track observed by the Mini-EUSO EM appears on 8 pixels for $\sim 160 \mathrm{~ms}$ ( $\sim 20 \mathrm{~ms} / \mathrm{pixel})$, its projection on the horizontal plane at $100 \mathrm{~km}$ altitude is $\sim 8 \mathrm{~km}$ long. Therefore, Mini-EUSO would have seen the same meteor event in 2 pixels for $\sim 80 \mathrm{~ms} /$ pixel.

If an event with the same observed characteristics of that in Fig. 11, i.e., crossing 8 pixels in $\sim 160 \mathrm{~ms}$, would be observed by Mini-EUSO from the ISS, the horizontal speed would be translated to $\sim 210 \mathrm{~km} / \mathrm{s}$ for a source at $\sim 100 \mathrm{~km}$ altitude. Such a high speed would be compatible with the speed of interstellar meteors, with origin outside the Solar System, that well exceed the escape velocity from the Sun. Such an event could be also interpreted as a nuclearite having a horizontal speed of $\sim 280 \mathrm{~km} / \mathrm{s}$ near the sea level, i.e., $\sim 400 \mathrm{~km}$ away from the ISS. For the Mini-EUSOlike detectors in the future, this could also be a space debris passing $\sim 10 \mathrm{~km}$ away at the apparent speed of $\sim 7 \mathrm{~km} / \mathrm{s}$. The angular speed and the light intensity would help to discriminate between possible object types.

\section{Conclusions}

The observations made with the Mini-EUSO EM offered an opportunity to test the prototype of the Mini-EUSO detector before it was launched and installed on the ISS in 2019. The tests described in this paper provided a large variety of data on different timescales, by which the detector performance was studied.

Tests at TurLab demonstrated that some improvements for the L1 trigger had to be performed, while the L2 trigger algorithm and the continuous D3 data acquisition were proved to work as expected. With a new system, which is basically a copy of the Mini-EUSO EM detector regarding the data acquisition and the processing, further studies were performed afterwards. A new threshold has been applied to the L1 trigger, that made it more robust and stronger against the non-standard background conditions (much lower or higher than $\sim 1$ count/pixel/D1 GTU) and was applied to the firmware of Mini-EUSO. Moreover, tests to estimate the reflectance of materials that compose space debris were performed, together with simulation analyses to study the capability of Mini-EUSO-like instruments to detect and track space debris from space.

Outdoor data acquisitions allowed to detect several natural and artificial light sources. Stars with observed apparent magnitude up to about +5.6 , already corrected for the atmospheric absorption, permitted a comparison between the performance of the Mini-EUSO EM and the PRISMA camera of the Astrophysical Observatory of Turin. The possible detection of meteors showed that the telescope is able to detect faint meteors with speeds of the order of tens of kilometers per second. The analysis of the night sky brightness at the zenith before sunrise showed that the signal in the UV band increased earlier than in the visible band, consistent with the fact that the UV photons are more scattered than the visible ones. Observations of artificial light from buildings showed the capability of detecting 
flashes from warning lights with frequencies of $\sim 0.7 \mathrm{~Hz}$ and the modulation of the electrical grid at $50 \mathrm{~Hz}$. Finally, the detection of a known space debris gave information to rescale the size and the distance to emulate the detection of space debris from space.

Observations of similar sources have been made by both the Mini-EUSO EM during the outdoor tests and Mini-EUSO from the ISS. This is the case for meteors, flashes, and the modulation of the alternating signal from the electrical grid.

The experience acquired with the engineering model before the Mini-EUSO launch allowed to predict some of the observations that can be made from space and to understand how to interpret them, for example using the modulation of the current to distinguish between artificial and natural light (such as bioluminescence). The results and studies discussed in this paper are important for the analysis and the operation of the Mini-EUSO telescope. Mini-EUSO already confirmed some expectations raised during the tests of the engineering model.

Acknowledgements The authors acknowledge all members of the JEM-EUSO Collaboration, especially the Mini-EUSO team. This work was supported by State Space Corporation ROSCOSMOS, by the Italian Space Agency through the ASI INFN agreement n. 2017-8-H.0 and contract n. 2016-1-U.0, by Basic Science Interdisciplinary Research Projects of RIKEN and JSPS KAKENHI grant (JP17H02905, JP16H02426 and JP16H16737), by the Italian Ministry of Foreign Affairs and International Cooperation, by the French space agency CNES, by the National Science Centre in Poland, grant 2017/27/B/ST9/02162 and grant 2020/37/B/ST9/01821. PRISMA was funded by 2016/0476 and 2019/0672 Research and Education grants from Fondazione Cassa di Risparmio di Torino and by a 2016 grant from Fondazione Agostino De Mari (Savona). The authors from the University of Turin acknowledge support from Compagnia di San Paolo within the project "ex-post-2018".

Funding Open access funding provided by Università degli Studi di Torino within the CRUI-CARE Agreement.

Open Access This article is licensed under a Creative Commons Attribution 4.0 International License, which permits use, sharing, adaptation, distribution and reproduction in any medium or format, as long as you give appropriate credit to the original author(s) and the source, provide a link to the Creative Commons licence, and indicate if changes were made. The images or other third party material in this article are included in the article's Creative Commons licence, unless indicated otherwise in a credit line to the material. If material is not included in the article's Creative Commons licence and your intended use is not permitted by statutory regulation or exceeds the permitted use, you will need to obtain permission directly from the copyright holder. To view a copy of this licence, visit http://creativecommons.org/licenses/by/4.0/.

\section{References}

1. Bacholle, S. et al.: Mini-EUSO mission to study Earth UV emissions on board the ISS. Astrophys. J. Suppl. S. 2, v.253 (2021)

2. Adams, J.H. Jr. et al., (JEM-EUSO Coll.): JEM-EUSO observational technique and exposure. Exp. Astron. 40, 117-134 (2015)

3. Adams, J.H.Jr. et al.: JEM-EUSO: Meteor And nuclearite observations. Exp. Astron. 40, 253-279 (2015)

4. Adams, J.H. et al.: The Jem-Euso instrument. Exp. Astron. 40, 19-44 (2015)

5. Ebisuzaki, T. et al.: Demonstration designs for the remediation of space debris from the International Space Station. Acta Astronaut. 112, 102-113 (2015)

6. Adams, J.H. et al.: Calibration for extensive air showers observed during the JEM-EUSO mission. Adv. Space Res. 53, 1506-1514 (2014) 
7. Abdellaoui, G. et al.: First observations of speed of light tracks by a fluorescence detector looking down on the atmosphere. J. Instrum. 13, P05023 (2018)

8. Kungel, V. et al., (JEM-EUSO Coll.): Laser test with Mini-EUSO. Proceedings of 36th International Cosmic Ray Conference PoS(ICRC2019)325 (2019)

9. TurLab. http://www.turlab.ph.unito.it/

10. Prieto-Alfonso, H. et al., (JEM-EUSO Coll.): Multi anode photomultiplier tube reliability assessment for the JEM-EUSO space mission. Proceedings of 33rd International Cosmic Ray Conference (2013)

11. Blin-Bondil, S. et al.: SPACIROC3: A Front-End Readout ASIC For JEM-EUSO cosmic ray observatory. Proceedings of Technology and Instrumentation in Particle Physics 2014 PoS(TIPP2014)172 (2014)

12. Capel, F., et al.: Mini-EUSO (Extreme Universe Space Observatory) data acquisition and control software. JATIS, v.5(4), 044009 (2019)

13. Belov, A. et al.: The integration and testing of the Mini-EUSO multi-level trigger system. Adv. Space Res. 62, 2966-2976 (2018)

14. Miyamoto, H. et al., (JEM-EUSO Coll.): The EUSO@Turlab Project. Proceedings of the XXV European Cosmic Ray Symposium (2016); arXiv:1701.07708

15. Suino, G. et al., (JEM-EUSO Coll.): The EUSO@Turlab Project: Results from Phase II. Proceedings of 35th International Cosmic Ray Conference PoS(ICRC2017)422 (2017)

16. Miyamoto, H. et al., (JEM-EUSO Coll.): The EUSO@Turlab: Test of Mini-EUSO Engineering Model, Proceedings of 36th International Cosmic Ray Conference PoS(ICRC2019)194 (2019)

17. Miyamoto, H. et al., (JEM-EUSO Coll.): Space debris detection and tracking with the techniques of cosmic ray physics. Proceedings of 36th International Cosmic Ray Conference PoS(ICRC2019)253 (2019)

18. Fenu, F. et al., (JEM-EUSO Coll.): Simulations for the JEM-EUSO program with ESAF. Proceedings of 36th International Cosmic Ray Conference PoS(ICRC2019)252 (2019)

19. MASTER ESA's software to assess the debris or meteoroid flux imparted on a spacecraft on an arbitrary Earth's orbit. https://sdup.esoc.esa.int/

20. Gardiol, D. et al:: News from the Italian PRISMA fireball network. Proceedings of the 37th International Meteor Conference, pp. 81-86 (2019)

21. Gardiol, D. et al.: Cavezzo, the first Italian meteorite recovered by the PRISMA fireball network. Orbit, trajectory, and strewn-field. Submitted to Mon. Not. R. Astron. Soc. 501, 1215-1227 (2021)

22. Barghini, D. et al.: Improving astrometry and photometry reduction for PRISMA all-sky cameras. Proceedings of the 37th International Meteor Conference, pp. 41-45 (2019)

23. Barghini, D. et al.: Astrometric calibration for all-sky cameras revisited. Astron. Astrophys. 626, A105 (2019)

24. Abdellaoui, G. et al.: Meteor studies in the framework of the JEM-EUSO program. Planet. Space Sci. 143, 245-255 (2017)

25. Falchie, F., et al.: Supplement to: The New World Atlas of Artificial Night Sky Brightness. V. 1.1. GFZ Data Services. The quoted value was given through the interface on https://www.lightpollutionmap. info/ (2016)

26. NOAA National Center for Environmental Information, Defense Meteorological Satellite Program (DMSP). https://ngdc.noaa.gov/eog/dmsp.html

27. Bisconti, F. et al., (JEM-EUSO Coll.): Mini-EUSO engineering model: tests in open-sky condition. Proceedings of 36th International Cosmic Ray Conference PoS(ICRC2019)198 (2019)

28. Analytical Graphics, Inc. Systems Tool Kit (STK). https://www.agi.com/products/stk

29. Flightradar24. https://www.flightradar24.com/

Publisher's note Springer Nature remains neutral with regard to jurisdictional claims in published maps and institutional affiliations. 


\section{Affiliations}

F. Bisconti ${ }^{1,2}$ (D) H. Miyamoto ${ }^{1,2} \cdot$ D. Barghini ${ }^{1,2,3}$ (D) $\cdot$ M. Battisti $i^{1,2}$ (D) $\cdot$ A. Belov ${ }^{4}$. M. E. Bertaina ${ }^{1,2}$ (D) . S. Blin-Bondil ${ }^{5,15}$. G. Cambiè 6,7 . F. Capel ${ }^{8,9}$. M. Casolino ${ }^{6,7,10}$ - A. Cellino ${ }^{2,3} \cdot$ L. Conti ${ }^{6,11}$. G. Contino ${ }^{12}$ (D) . G. Cotto ${ }^{1,2}$.

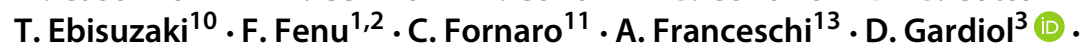
A. Haungs ${ }^{14}$ (D) P. Klimov ${ }^{4}$ (D) M. Manfrin ${ }^{1,2} \cdot$ L. Marcelli $^{6} \cdot$ M. Mignone ${ }^{2}$. T. Napolitano ${ }^{13}$. E. Parizot ${ }^{15}$ (D) P. Picozza ${ }^{6,7}$. L. W. Piotrowski ${ }^{16,10}$. G. Prévôt ${ }^{15} \cdot$ E. Reali,7,7 M. Ricci ${ }^{13}$ D $\cdot$ K. Shinozaki ${ }^{1,17} \cdot$ F. Simioli ${ }^{11}$ - G. Suino ${ }^{1,2}$. J. Szabelski ${ }^{17}$

\section{H. Miyamoto} hiroko.miyamoto@to.infn.it

1 Department of Physics, University of Turin, Turin, Italy

2 Section of Turin, National Institute for Nuclear Physics, Turin, Italy

3 Astrophysical Observatory of Turin, National Institute for Astrophysics, Turin, Italy

4 Skobeltsyn Institute of Nuclear Physics, Lomonosov Moscow State University, Moscow, Russia

5 OMEGA, Ecole Polytechnique, Palaiseau, France

6 Section of Roma Tor Vergata, National Institute for Nuclear Physics, Rome, Italy

7 Department of Physics, University of Roma Tor Vergata, Rome, Italy

8 Technical University of Munich, Munich, Germany

9 KTH Royal Institute of Technology, Stockholm, Sweden

10 RIKEN, Wako, Japan

11 International Telematic University Uninettuno, Rome, Italy

12 Institute of Space Astrophysics and Cosmic Physics, National Institute for Astrophysics, Palermo, Italy

13 Frascati National Laboratory, National Institute for Nuclear Physics, Rome, Italy

14 Institute for Astroparticle Physics, Karlsruhe Institute of Technology, Karlsruhe, Germany

15 Université de Paris, CNRS, Astroparticule et Cosmologie, F-75013 Paris, France

16 University of Warsaw, Warsaw, Poland

17 National Centre for Nuclear Research, Lodz, Poland 